\title{
Time-Depentent Hartree-Fock description of heavy ions fusion
}

\author{
Cédric Simenel and Benoît Avez \\ $D S M / D A P N I A / S P h N, C E A / S A C L A Y$, \\ F-91191 Gif-sur-Yvette Cedex, France \\ cedric.simenel@cea.fr
}

(Dated: December 17, 2018)

\begin{abstract}
A microscopic mean-field description of heavy ions fusion is performed in the framework of the Time-Dependent Hartree-Fock (TDHF) theory using a Skyrme interaction with the SLy4d parametrization. A good agreement with experiments is obtained on the position of the fusion barriers for various total masses, mass asymmetries and deformations. The excitation function of the ${ }^{16} \mathrm{O}+{ }^{208} \mathrm{~Pb}$ is overestimated by about $16 \%$ above the barrier. The restriction to an independent particles state in the mean-field dynamics prevents the description of sub-barrier fusion. Effect of transfer on fusion is discussed.
\end{abstract}

\section{INTRODUCTION}

Description of nuclear reactions is very challenging, especially at energies around the fusion barrier generated by the competition between Coulomb and nuclear interactions. It has been established that the structure of the collision partners may affect strongly the reaction mechanisms in this energy domain, as, for instance, the fusion cross-sections (for a review, see e.g. Ref. [1]).

Upcoming exotic beams facilities at energies of a few $\mathrm{MeV} / \mathrm{u}$ like SPIRAL2 will allow studies of the interplay between reaction mechanisms and exotic nuclear structures such as haloes, neutron skins, high isospins... It is therefore recommended to treat both structure and dynamics within the same formalism. This is the case of fully microscopic approaches such as the Time-Dependent Hartree-Fock (TDHF) theory proposed by P. A. M. Dirac in 1930 [2]. This time dependent version of the well known Hartree-Fock (HF) theory gives a self-consistent mean-field description of nuclear dynamics $[3,4]$. The success of the first HF calculations based on the Skyrme interaction $[5,6]$ led to tremendous activities to describe nuclear structure within mean-field based approaches (see Ref. [7] for a review).

Early TDHF calculations has been done with the seek for a description of the dynamics of nuclei as good as their static properties [8-16]. They used various symmetries and simplified Skyrme interactions to reduce the computational time. The increase of computational power allowed realistic TDHF calculations of nuclear collisions in 3 dimensions with full Skyrme interactions in the last ten years [17-23].

In this paper, we present a TDHF study of nuclear fusion. In a first part, we recall the formalism and detail the calculation. In a second part, we calculate fusion barriers for several systems with various total masses and mass asymmetries and compare them to experimental data. We also consider the case where one of the collision partners is prolately deformed. Finally, we calculate the excitation function for the total fusion cross section of the system ${ }^{16} \mathrm{O}+{ }^{208} \mathrm{~Pb}$ and compare to experiments before to conclude.

\section{THE TIME-DEPENDENT HARTREE-FOCK APPROACH}

\section{A. Formalism}

Let us first recall some aspects of the TDHF theory. In a non relativistic microscopic approach, the system is described by a N-particles state $|\psi\rangle$ which is solution of the Schroedinger equation

$$
i \hbar \frac{\partial}{\partial t}|\psi\rangle=\hat{H}|\psi\rangle
$$

with the microscopic Hamiltonian ${ }^{1}$

$$
\hat{H}=\sum_{i=1}^{N} \frac{\hat{p}(i)^{2}}{2 m}+\sum_{i>j=1}^{N} \hat{v}(i, j) .
$$

\footnotetext{
${ }^{1}$ We consider only two-body interactions.
} 
The state $|\psi\rangle$ contains all the information on the system, which is more than what we really need for a good description of the dynamics. We often need only expectation values of one-body observables, such as the position of the fragments, their shapes and particle numbers. These quantities are determined from the one-body density matrix $\rho$ with elements $\rho_{i j}=\left\langle\psi\left|\hat{a}_{j}^{\dagger} \hat{a}_{i}\right| \psi\right\rangle$. The expectation value of a one-body observable $\hat{F}=\sum_{i j} f_{i j} \hat{a}_{i}^{\dagger} \hat{a}_{j}$ is then given by $\langle\psi|\hat{F}| \psi\rangle=\operatorname{Tr}(\rho f)$.

The first step toward the TDHF theory is to restrict the description to one-body observables, and to seek for an equation giving the evolution of $\rho$. Starting from Eq. (1) and using the Bogolyubov-Born-Green-Kirkwood-Yvon (BBGKY) hierarchy [24-26], we can show that the one-body density matrix follows [27]

$$
i \hbar \frac{\partial}{\partial t} \rho=[h[\rho], \rho]+\operatorname{Tr}_{2}[v(1,2), C(1,2)]
$$

where $h[\rho]$ is the HF single-particle Hamiltonian with matrix elements $h_{i j}=\langle i|\hat{h}[\rho]| j\rangle=\frac{\delta}{\delta \rho_{j i}}\langle\psi|\hat{H}| \psi\rangle$ and $C$ is the correlated part of the two-body density matrix.

Eq. (3) is exact but has two unknown quantities: $\rho$ and $C$. The second step toward the TDHF equation is to neglect the second term of the right hand side in Eq. (3). This can be done in two alternative ways:

- The correlation $C$ vanishes if we impose $|\psi\rangle$ to be an independent particles state at any time. The variational principle $\delta\left[\int \mathrm{d} t\left\langle\psi\left|\hat{H}-i \hbar \frac{\partial}{\partial t}\right| \psi\right\rangle\right]=0$, which is equivalent to Eq. (1), is then solved in the subspace of Slater determinants.

- The truncation of the BBGKY hierarchy can also be done by neglecting the residual interaction $\hat{V}_{\text {res }}=\hat{H}-$ $\sum_{i=1}^{N} \hat{h}[\rho](i)$. This is a mean-field approximation because the Hamiltonian is approximated by a one-body operator $\hat{H} \simeq \sum_{i j} h_{i j} \hat{a}_{i}^{\dagger} \hat{a}_{j}$. In this case, a system described by a Slater determinant at an initial time will be an independent particles state at any time.

We finally get the TDHF equation $i \hbar \frac{\partial}{\partial t} \rho=[h[\rho], \rho]$ where $\rho$ is now the one-body density matrix of an independent particles state. The operator associated to $\rho$ acts in the Hilbert space of single-particle states. It is written $\hat{\rho}=$ $\sum_{i=1}^{N}\left|\varphi_{i}\right\rangle\left\langle\varphi_{i}\right|$ where $\left|\varphi_{i}\right\rangle$ denotes an occupied single-particle state.

The TDHF theory neglects the pairing correlations which are contained in $C$. In fact, TDHF describes the evolution of occupied single-particle wave functions in the mean field generated by all the particles and assures an exact treatment of the Pauli principle during the dynamics.

\section{B. Practical aspects}

The advantage of TDHF is that it treats static properties and dynamics of nuclei within the same formalism and the same interaction. The initial state is obtained through static HF calculations which reproduce well nuclear binding energies and deformations. TDHF can be used in two ways for nuclear reactions:

- A single nucleus is evolved in an external field [8], simulating for instance the Coulomb field of the collision partner [28].

- The evolution of two nuclei, initially with a zero overlap, is represented by a single Slater determinant [9, 16].

The first case is well suited for inelastic scattering, like Coulomb excitation of vibrational and rotational states. The second case is used for more violent collisions like fusion reactions. In the latter, the lack of a collision term might be a drawback. At low energy, however, fusion is driven by one-body dissipation because the Pauli blocking prevents nucleon-nucleon collisions. Fusion occurs by transferring relative motion into internal excitation via one-body mechanisms well treated by TDHF.

Another important advantage of TDHF for near-barrier reaction studies is that it contains all types of couplings between the relative motion and internal degrees of freedom whereas in coupled channels calculations one has to include them explicitly according to physical intuition. The symmetries corresponding to the internal degrees of freedom of interest have to be relaxed in TDHF. However, it gives only classical trajectories for the time-evolution and expectation values of one-body observables. In particular, it does not include tunneling of the global wave function. 
We use the TDHF3D code built by P. Bonche and coworkers with the SLy4d Skyrme parametrization [17] which is a variant of the SLy4 one [29] specifically designed for TDHF calculations ${ }^{2}$. This code has a plane of symmetry (the collision plane). It uses the Skyrme energy functional expressed in Eq. (A.2) of Ref. [30] where the tensor coupling between spin and gradient has been neglected. The step size of the network is $0.8 \mathrm{fm}$ and the step time $0.45 \mathrm{fm} / \mathrm{c}$.

A TDHF calculation of two colliding nuclei is performed assuming that the two collision partners are initially at a distance $D_{0}$ in their HF ground state. This distance has to be big enough to allow Coulomb excitation in the entrance channel (polarization, vibration, rotation...). This initial distance is chosen to be $D_{0}=44.8 \mathrm{fm}$. We assume that before to reach this distance, the nuclei followed a Rutherford trajectory, which determines their initial velocities $\mathbf{v}_{1}$ and $\mathbf{v}_{2}$. The Galilean transformation $\hat{\rho}_{i}(t=0)=e^{i m \mathbf{v}_{i} \cdot \hat{\mathbf{r}}} \hat{\rho}_{H F_{i}} e^{-i m \mathbf{v}_{i} \cdot \hat{\mathbf{r}}}$ applied on the HF density matrix of the nucleus $i(i=1$ or 2$)$ put it into motion with the velocity $\mathbf{v}_{i}[31]$.

\section{FUSION BARRIERS}

Let us consider the simple case of fusion barriers. They are classically defined as the energy threshold above which fusion occurs for a head-on collision. Experimentally, the average position of the barrier can be approximated by the centroid of the so-called barrier distribution $D_{B}(E)=\frac{\mathrm{d}^{2}}{\mathrm{~d}^{2}}\left(\sigma_{f u s}(E) E\right)$ where $\sigma_{f u s}$ is the fusion cross section [32]. In the special case of a single barrier for a classical system, the barrier distribution is a Dirac distribution $D_{B} \sim \delta(E-B)$, whereas a width is generated by tunneling for a quantum system. Several barriers may be generated by the coupling between internal degrees of freedom and the relative motion [1].

To determine the fusion barrier from TDHF, we consider head-on collisions at various energies. The barrier is then located between the highest energy for which there is no fusion and the lowest one for which fusion occurs. The average barrier is first studied for spherical nuclei. Then the case of deformed nuclei is considered.

\section{A. Spherical nuclei}

Fig. 1 shows the density plot for a ${ }^{16} \mathrm{O}+{ }^{208} \mathrm{~Pb}$ central collision at a center of mass energy $E=74.44 \mathrm{MeV}$. After a neck formation, the system separates into two fragments. Fig. 2 shows the same reaction at $E=74.45 \mathrm{MeV}$. We see that adding $10 \mathrm{keV}$ is enough to fuse. We deduce the fusion barrier $V_{B}^{T D H F}=74.445 \pm 0.005 \mathrm{MeV}$. This value is in excellent agreement with the experimental one $V_{B}^{\text {exp. }} \simeq 74.5 \mathrm{MeV}$ [33]. It is interesting to note that, if we assume frozen $\mathrm{HF}$ densities of the collision partners obtained with the same interaction, we get a barrier $V_{B}^{\text {frozen }}=76.55 \mathrm{MeV}$ at a radius $R_{B}^{\text {frozen }}=11.73 \mathrm{fm}$. These values are close to the Bass barrier $V_{B}^{\text {Bass }}=77.10 \mathrm{MeV}$ at $R_{B}^{\text {Bass }}=11.42 \mathrm{fm}$ $[34,35]$, but overestimate the experimental fusion barrier. We conclude that the TDHF calculations contain dynamical effects that reduce the barrier by $\sim 2 \mathrm{MeV}$ as compared to the frozen approximation. It has been suggested that transfer may affect the barrier for this system [33]. Indeed, we see in Fig. 2 that the two fragments are linked by a neck and form a di-nuclear system during $\sim 400 \mathrm{fm} / \mathrm{c}$. It is enough time for the nuclei to transfer nucleons, leading to a dynamical evolution of the barrier.

To get a deeper insight into this transfer preceding fusion, we focus on the case just below the barrier (Fig. 1). Here, almost two protons and no neutron, in average, have been transfered from the ${ }^{16} \mathrm{O}$ to the ${ }^{208} \mathrm{~Pb}$. The two-protons transfer from the light to the heavy nucleus is then expected to be an important channel at the barrier. This is consistent with experimental observations of relatively high Carbon production cross sections, of the same order of the Nitrogen ones, in the exit channel of ${ }^{16} \mathrm{O}+{ }^{208} \mathrm{~Pb}$ at the barrier [36, 37].

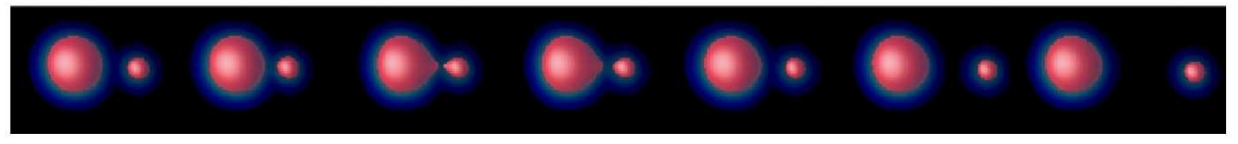

FIG. 1: Densities associated to a ${ }^{16} \mathrm{O}+{ }^{208} \mathrm{~Pb}$ central collision at a center of mass energy $E=74.44 \mathrm{MeV}$. The surface corresponds to an isodensity at half the saturation density. Each plot is separated by $135 \mathrm{fm} / \mathrm{c}$.

\footnotetext{
2 The calculations are performed in the laboratory frame and not in the intrinsic frame. It is then necessary to remove the center of mass correction in the fitting procedure of the interaction and in the initial HF calculations of the collision partners. The " $d$ " in SLy $4 d$ stands for dynamics.
} 


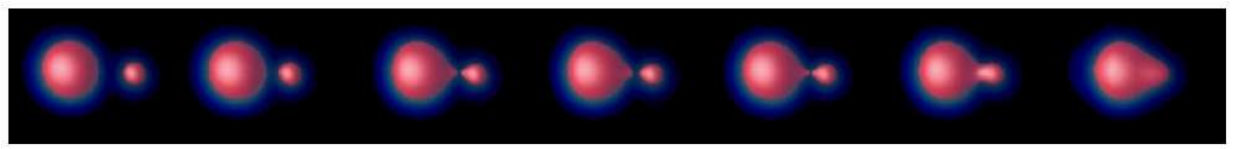

FIG. 2: Same as Fig. 1 at $E=74.45 \mathrm{MeV}$.

Finally, Fig. 3 shows a comparison between experimental fusion barriers and those from TDHF calculations for systems with various total masses and mass asymmetries. The lowest barrier is for ${ }^{40} \mathrm{Ca}+{ }^{40} \mathrm{Ca}$ and the highest one for ${ }^{48} \mathrm{Ti}+{ }^{208} \mathrm{~Pb}$. The agreement is of the same order than with the Bass barrier [34, 35]. Remembering that TDHF has no adjustable parameter on reaction mechanisms, we conclude that one can use it for fusion barriers prediction with confidence.

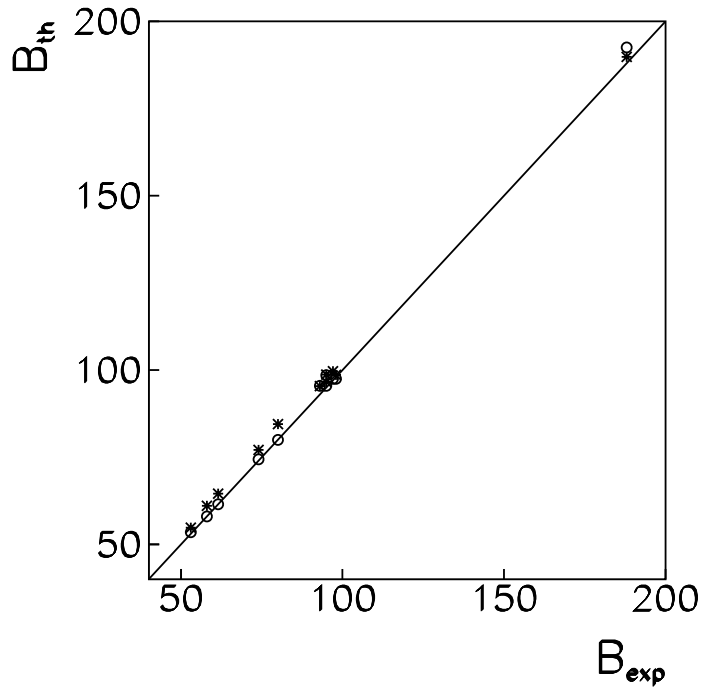

FIG. 3: Theoretical fusion barriers from TDHF calculations (circles) and the Bass barriers (stars) as function of the experimental values (from barrier distributions centroid).

\section{B. Effect of deformation on fusion}

We now consider collisions of a spherical nucleus on a deformed one. In such a case, the barrier depends on the orientation of the deformed nucleus at the touching point, leading to a wider barrier distribution than the single barrier case [1].

\section{Heavy deformed + light spherical nuclei}

Fig. 4 shows barrier distributions for collisions with a light spherical projectile $\left({ }^{16} \mathrm{O}\right)$ on heavy prolately deformed targets ${ }^{154} \mathrm{Sm}$ (left) and ${ }^{238} \mathrm{U}$ (right) $[38,39]$. Their width are $\sim 7-10 \mathrm{MeV}$ and cannot be explained by tunneling alone. The latter adds a width of only $2-3 \mathrm{MeV}$ [32]. Such barrier distributions are usually well reproduced in the framework of coupled channel calculations [1]. In addition, microscopic theories like TDHF can help to understand the physics process generating these couplings.

The barriers predicted by the TDHF calculations for these systems are also shown (arrows) on Fig. 4 for two extreme configurations of central collisions where the collision and deformation axis are either parallel or perpendicular. In the parallel configuration, the Coulomb repulsion is smaller and so is the resulting barrier. All the intermediate orientations at the touching point give fusion barriers between the parallel and perpendicular configurations ones. In addition to a good reproduction of the centroid, the TDHF calculations also reproduce the width of the barrier distributions generated by a static deformation of the target without any adjustment of parameters. 


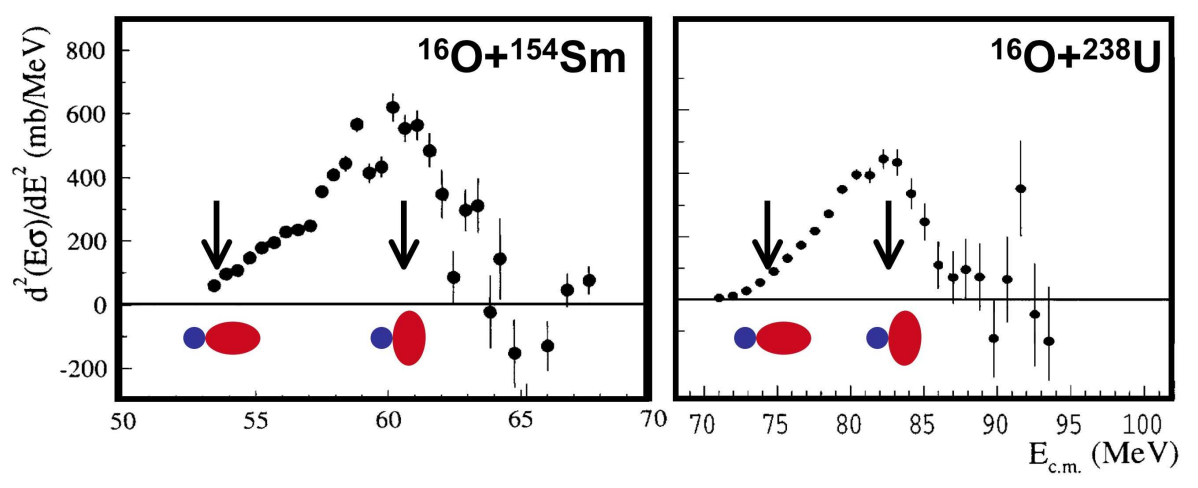

FIG. 4: Experimental barrier distributions for ${ }^{16} \mathrm{O}+{ }^{154} \mathrm{Sm}$ (left) and ${ }^{16} \mathrm{O}+{ }^{238} \mathrm{U}$ (right). The arrows indicate the barriers obtained from TDHF calculations for two extreme configurations where the collision and deformation axis are parallel (lowest barriers) and perpendicular (highest barriers).

The shape of these distributions is due to a prolate deformation of the target: the distribution is more peaked at higher energies. The configuration where the collision and deformation axis are parallel corresponds to only one possible orientation, whereas the perpendicular configuration can be reached by any orientation for which the deformation axis is contained in the plane perpendicular to the collision axis. If one assumes an isotropic distribution of the orientations at the touching point, which is a reasonable approximation when the spherical nucleus is light and the deformed one heavy [28], then the perpendicular configuration is more probable than the parallel one and the barrier distribution is peaked at high energies. One gets the opposite with an oblate nucleus instead of a prolate one.

\section{2. light deformed + heavy spherical nuclei}

The case of a light deformed projectile on a heavy spherical target has been investigated theoretically in Ref. [28] both within the TDHF and the coupled channel frameworks with the code CCFULL [40]. For such systems, the barrier distribution gets affected due to reorientation of the deformed nucleus in the Coulomb field of the target. This breaks the isotropy of the orientation axis distribution and results to a fusion hindrance at low energies. Experimental evidences of this effect have been reported recently [41]. The reorientation is proportional to $A_{\text {spherical }} / A_{\text {total }}$ and then does not affect the systems studied in Fig. $4^{3}$.

\section{EXCITATION FUNCTION OF ${ }^{16} \mathrm{O}+{ }^{208} \mathrm{~PB}$}

We now focus on fusion cross sections given by

$$
\sigma_{f u s}(E)=\frac{\pi \hbar^{2}}{2 \mu E} \sum_{l=0}^{\infty}(2 l+1) P_{f u s}(E, l)
$$

where $\mu$ is the reduced mass of the system and $P_{f u s}(E, l)$ is the fusion probability at a center of mass energy $E$ and an angular momentum $\sqrt{l(l+1)} \hbar$. The restriction to an independent particles state, as in TDHF, leads to $P_{\text {fus }}=1$ for $l \leq l_{\max }$ and 0 for $l>l_{\max }$. We then get the so-called "quantum sharp cut-off formula" [42]

$$
\sigma_{f u s}(E)=\frac{\pi \hbar^{2}}{2 \mu E}\left(l_{\max }+1\right)^{2} .
$$

To avoid discontinuities introduced by the cut-off and the integer nature of $l_{\max }$, we approximate $\left(l_{\max }+1\right) \hbar$ by its classical equivalent $\mathcal{L}_{c}$. The latter is the threshold of the classical angular momentum $\mathcal{L}=\sqrt{2 \mu E} b, b$ being the impact

\footnotetext{
3 Though it is a Coulomb effect, the reorientation does not depend on the charges of the nuclei but on their masses as we can see in Eq. (5) of Ref. [28].
} 
parameter, below which fusion occurs [35]. This approximation is justified by the fact that both $\left(l_{\text {max }}+1\right)^{2}$ and $\mathcal{L}_{c}^{2} / \hbar^{2}$ are greater than $l_{\max }\left(l_{\max }+1\right)$ and smaller than $\left(l_{\max }+1\right)\left(l_{\max }+2\right)$. We finally get the classical expression for the fusion cross section $\sigma_{\text {fus }}(E) \simeq \pi \mathcal{L}_{c}^{2} / 2 \mu E$.

Figure 5 shows the excitation function obtained for the ${ }^{16} \mathrm{O}+{ }^{208} \mathrm{~Pb}$ system in comparison to experimental data [33]. There is a good agreement above the barrier, though the fusion cross sections are overestimated by about $16 \%$. However, the fusion cross section vanishes below the barrier, following a classical behavior. This drawback of TDHF is well known and is due to the restriction to a single independent particles state. Indeed, to get a fusion probability between 0 and 1, we need at least two Slater determinants: one describing the two well separated fragments after the collision when fusion does not occur and one describing the fused system. It is necessary to go beyond TDHF to treat a sum of Slater determinants and then to describe sub-barrier fusion due to tunneling effects.

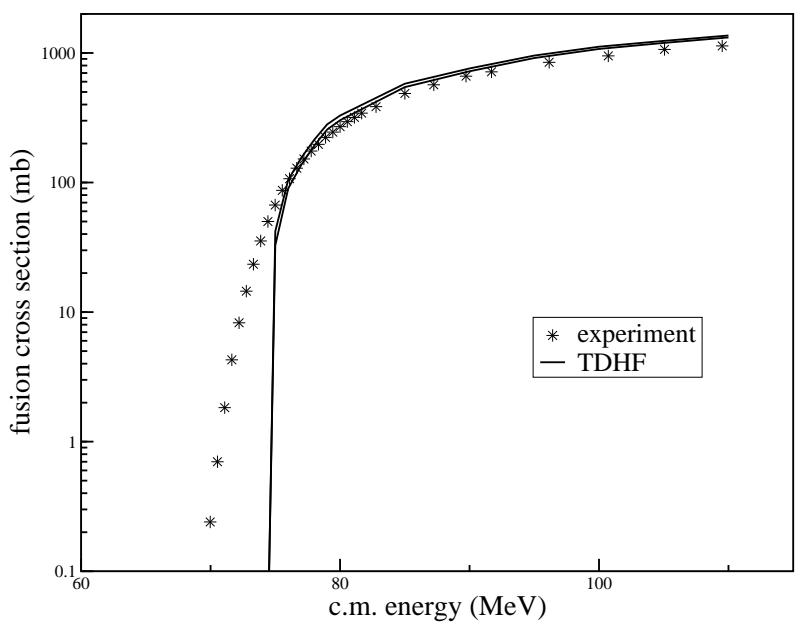

FIG. 5: Experimental fusion excitation function (stars) for ${ }^{16} \mathrm{O}+{ }^{208} \mathrm{~Pb}$. The lines denote the upper and lower limits for the fusion cross sections obtained from TDHF calculations.

\section{CONCLUSIONS AND PERSPECTIVES}

We presented some applications of the Time-Dependent Hartree-Fock theory to nuclear fusion. The only phenomenological input is the set of parameters of the SLy4d force which have not been adjusted on any reaction mechanism like cross sections or fusion barriers for instance. Despite this, the agreement between the TDHF calculations and the experimental fusion barriers is excellent for a wide range of projectiles and targets. The width of fusion barrier distributions generated by a static deformation of the target is also well reproduced.

The TDHF calculations overestimate the fusion cross sections for the system ${ }^{16} \mathrm{O}+{ }^{208} \mathrm{~Pb}$ above the barrier by about $16 \%$. Below the barrier, the fusion cross section vanishes in TDHF calculations. The sub-barrier fusion due to quantum tunneling of the many-body wave function is not present in TDHF. This is due to the restriction to a single independent particles state.

Though the fusion cross section has a classical behavior, the quantum nature of the single-particle wave functions is well treated. Thus, it would be interesting to study the transfer of nucleons from one nucleus to the other within $\mathrm{TDHF}^{4}$. An illustration of such transfer is seen in the TDHF calculation of the ${ }^{16} \mathrm{O}+{ }^{208} \mathrm{~Pb}$ just below the barrier.

\section{Acknowledgements}

We thank P. Bonche who provided his TDHF code and initiated this work. We are also grateful to D. Lacroix, M. Bender, K. Bennaceur, Ph. Chomaz and T. Duguet for fruitful discussions. The calculations have been performed in

\footnotetext{
${ }^{4}$ We have to distinguish between the transfer of typically one or two nucleons and more violent collisions like deep-inelastic reactions. In the latter, the width of the particle number distribution is known to be underestimated with a single Slater determinant [43].
} 
the Centre de Calcul Recherche et Technologie of the Commisariat à l'Énergie Atomique.

[1] M. Dasgupta, D. J. Hinde, N. Rowley and A. M. Stefanini, Annu. Rev. Nucl. Part. Sci. 48 (1998) 401 and ref. therein.

[2] P. A. M. Dirac, Proc. Camb. Phil. Soc. 26 (1930) 376.

[3] D. R. Hartree, Proc. Camb. Phil. Soc. 24 (1928) 89.

[4] V. A. Fock, Z. Phys. 61 (1930) 126.

[5] T. Skyrme, Phil. Mag. 1 (1956) 1043.

[6] D. Vautherin and D. M. Brink, Phys. Rev. C5 (1972) 626.

[7] M. Bender, P.-H. Heenen and P.-G. Reinhard, Rev. Mod. Phys. 75 (2003) 121.

[8] Y. M. Engel et al., Nucl. Phys. A249 (1975) 215.

[9] P. Bonche, S. Koonin and J. W. Negele, Phys. Rev. C13 (1976) 1226.

[10] P. Bonche, B. Grammaticos and S. Koonin, Phys. Rev. C17 (1978) 1700.

[11] H. Flocard, S. E. Koonin and M. S. Weiss, Phys. Rev. C17 (1978) 1682.

[12] S. J. Krieger and K. T. R. Davis, Phys. Rev. C18 (1978) 2567.

[13] K. T. R. Davies, V. Maruhn-Rezwani, S. E. Koonin and J. W. Negele, Phys. Rev. Lett. 41 (1978) 632.

[14] K. R. S. Devi, A. K. Dhar and M. R. Strayer, Phys. Rev. C23 (1981) 2062.

[15] S. J. Krieger and M. S. Weiss, Phys. Rev. C24 (1981) 928.

[16] J. W. Negele, Rev. Mod. Phys. 54 (1982) 913.

[17] K.-H. Kim, T. Otsuka and P. Bonche, J. Phys. G23 (1997) 1267.

[18] C. Simenel, Ph. Chomaz and G. de France, Phys. Rev. Lett. 86 (2001) 2971.

[19] T. Nakatsukasa and K. Yabana, Phys. Rev. C71 (2005), 024301.

[20] A. S. Umar and V. E. Oberacker, Phys. Rev. C73 (2006) 054607; Phys. Rev. C74 (2006) 021601(R); Phys. Rev. C74 (2006) 024606; Phys. Rev. C74 (2006) 061601; Phys. Rev. C76 (2007) 014614.

[21] J. A. Maruhn, P.-G. Reinhard, P. D. Stevenson and M. R. Strayer, Phys. Rev. C74 (2006) 027601.

[22] L. Guo, J. A. Maruhn and P.-G. Reinhard, Phys. Rev. C76 (2007) 014601.

[23] C. Simenel, Ph. Chomaz and G. de France, Phys. Rev. C76 (2007) 024609.

[24] N. N. Bogolyubov, J. Phys. (URSS) 10 (1946) 256.

[25] H. Born and H. S. Green, Proc. Roy. Soc. A188 (1946) 10.

[26] J. G.Kirwood, J. Chem. Phys. 14 (1946) 180.

[27] D. Lacroix, S. Ayik and Ph. Chomaz, Prog. Part. Nucl. Phys. 52 (2004) 497.

[28] C. Simenel, Ph. Chomaz and G. de France, Phys. Rev. Lett. 93 (2004) 102701.

[29] E. Chabanat et al., Nucl. Phys. A635 (1998) 231.

[30] P. Bonche, H. Flocard and P.-H. Heenen, Nucl. Phys. A467 (1987) 115.

[31] D. J. Thouless and J. G. Valatin, Nucl. Phys. 31 (1962) 211.

[32] N. Rowley, G. R. Satchler and P. H. Stelson, Phys. Lett. B254 (1991) 25.

[33] C. R. Morton et al., Phys. Rev. C60 (1999) 044608.

[34] R. Bass, Phys. Rev. Lett. 39 (1977) 265.

[35] R. Bass, Nuclear Reactions (Springer-Verlag, Berlin, 1980).

[36] F. Videbæk et al., Phys. Rev. C15 (1977) 954.

[37] E. Vulgaris, L. Grodzins, S. G. Steadman and R. Ledoux, Phys. Rev. C33 (1986) 2017.

[38] J. R. Leigh et al., Phys. Rev. C52 (1995) 3151.

[39] D. J. Hinde et al., Phys. Rev. C53 (1996) 1290.

[40] K. Hagino, N. Rowley and A. T. Kruppa, Comp. Phys. Com. 123 (1999) 143.

[41] B. K. Nayak et al., Phys. Rev. C75 (2007) 054615.

[42] J. S. Blair, Phys. Rev. 95 (1954) 1218.

[43] C. H. Dasso, T. Døssing and H. C. Pauli, Z. Phys. A289 (1979) 395. 